\title{
Functional Micro-Imaging of Soft and Hard Tissue using Synchrotron Light
}

\author{
Philipp J. Thurner ${ }^{\mathrm{a}, \mathrm{b}}$, Peter Wyss ${ }^{\mathrm{a}}$, Romain Voide ${ }^{\mathrm{b}}$, Martin Stauber ${ }^{\mathrm{b}}$, Bert Müller ${ }^{\mathrm{b}}$, \\ Marco Stampanoni, ${ }^{b}$, Jeffrey A. Hubbell ${ }^{\mathrm{d}}$, Ralph Müller ${ }^{\mathrm{b}}$, and Urs Sennhauser ${ }^{\mathrm{a}}$ \\ ${ }^{a}$ Swiss Federal Laboratories for Materials Testing and Research (EMPA), Dübendorf Switzerland \\ ${ }^{\mathrm{b}}$ Swiss Federal Institute of Technology Zürich (ETHZ), Zürich, Switzerland \\ ${ }^{c}$ Swiss Light Source (SLS), Paul Scherrer Institute (PSI), Villigen, Switzerland \\ ${ }^{\mathrm{d}}$ Swiss Federal Institute of Technology Lausanne (EPFL), Lausanne, Switzerland
}

\begin{abstract}
In current biological and biomedical research, quantitative endpoints have become an important factor of success. Classically, such endpoints were investigated with 2D imaging, which is usually destructive and the 3D character of tissue gets lost. 3D imaging has gained in importance as a tool for both, qualitative and quantitative assessment of biological systems. In this context synchrotron radiation based tomography has become a very effective tool for opaque 3D tissue systems. Cell cultures and adherent scaffolds are visualized in 3D in a hydrated environment, even uncovering the shape of individual cells. Advanced morphometry allows to characterize the differences between the cell cultures of two distinct phenotypes. Moreover, a new device is presented enabling the 3D investigation of trabecular bone under mechanical load in a time-lapsed fashion. Using the highly brilliant X-rays from a synchrotron radiation source, bone microcracks and an indication for un-cracked ligament bridging are uncovered. 3D microcrack analysis proves that the classification of microcracks from 2D images is ambiguous. Fatigued bone was found to fail in burstlike fashion, whereas non-fatigued bone exhibited a distinct failure band. Additionally, a higher increase in microcrack volume was detected in fatigued in comparison to non-fatigued bone. The developed technologies prove to be very effective tools for advanced 3D imaging of both hard and soft tissue.
\end{abstract}

Keywords: Imaging, Image Processing, Micro-Computed Tomography, Synchrotron Radiation, Soft Tissue, Cell Cultures, Contrast Agents, Bone, Image Guided Failure Assessment, Microdamage

\section{INTRODUCTION}

Quantitative endpoints have become the important factor of success in basic aspects of biology and biomedical engineering; therefore, the 3D imaging has become increasingly important. It is often the basis on which both qualitative (visualization) and quantitative (morphometry) image processing is performed. In this context, X-ray tomography is a very promising tool. It can be used at different hierarchical scales in terms of specimen size and spatial resolution. Contrary to bone tissue, which is extensively studied with micro-computed tomography $(\mu \mathrm{CT})$ and synchrotron radiation based $\mu \mathrm{CT}(\mathrm{SR} \mu \mathrm{CT})$, the absorption differences for soft tissue with spatial resolution in the micrometer range are far to be resolved. Classical 2D imaging techniques have been used to study tissue and cell cultures at this level of spatial resolution and even below this level. Light microscopy (LM) and electron microscopy (EM) are typical examples which are mainly based on staining and labeling the features of interest (1). These samples include 2D cell cultures; as well as, histological sections from 3D tissue. It is, however, generally accepted that 3D cell cultures better resemble biological tissue than their 2D counterparts. Additionally, the investigations of these systems have been mainly of phenomenological nature. Nevertheless, the need for the comprehensive morphometric analysis is growing. This is mainly spurred by results from experiments that show a large difference in cell response in 2D and 3D cultures, respectively (2). It is clear that the systematic approach in modern biology is increasingly changing from $2 \mathrm{D}$ to $3 \mathrm{D}$ experiments in order to get closer to the in vivo situation. Thus, related imaging techniques are needed for the nondestructive 3D characterization of individual cells and cell cultures. Although confocal laser scanning microscopy (CLSM) is a method widely used for 3D cell imaging, it has some limitations that make the retrieval of true geometric data in all three dimensions rather difficult $(3,4)$. CLSM is also restricted to a sample depth of about $0.5 \mathrm{~mm}$ and to optically transparent samples. Alternatively, tomography using X-rays is a well established technique in materials science and bone characterization that is also applied to extended systems like body parts or whole organs of human beings (5) and to systems of nanometer scale such as individual biological cells (6) using the same principles at 
different levels of spatial resolution. Consequently, SR $\mu \mathrm{CT}$ allows investigations of cells and cell clusters, and their incorporation into tissues and organs in a hierarchical fashion - from human, to organ, to tissue and down to the individual cell - from the meter to the nanometer scale over more than 6 orders of magnitude. Moreover, the volumetric nature of SR $\mu \mathrm{CT}$ allows the quantitative assessment of cells and cell cultures. Last but not least, SR $\mu \mathrm{CT}$ is nondestructive allowing multiple time-lapsed imaging of samples. This is especially important for functional imaging of tissue related to biomechanics and functional adaptation of cells to mechanical loading and displacement, referred to as mechanobiology.

Bone belongs to the best investigated biological materials due to its primary function of providing skeletal stability and load carrying capacity. Bone is susceptible to different local stimuli including mechanical forces and has great capabilities in adapting its mechanical properties to the changes in its environment. Nevertheless, aging or hormonal changes make the bone weak, and it is loosing its ability to appropriate remodeling. Therefore, bone research is primarily motivated by the social impact and the immense costs in health care associated with osteoporosis $(7,8)$. Osteoporosis results in bone loss and deterioration in trabecular architecture causing the decrease of bone strength, and the concomitant increase in fracture risk. Due to the emergence of accurate and precise bone densitometry over the last two decades, bone density has been a primary endpoint in osteoporosis diagnosis and monitoring. Where strong correlations between bone density and mechanical properties of trabecular bone have been demonstrated for large populations using power-law regressions (9-15), changes in density can explain only $10 \%-90 \%$ variation of trabecular bone strength on an individual basis (16), leaving sometimes up to $90 \%$ of the strength variation unexplained. Thus, accurate diagnosis in a clinical environment based solely on bone densitometry proves to be difficult. The relative contribution of bone density, microarchitecture and local tissue properties, a complex referred to as bone "quality", to the mechanical stability of bone is poorly understood. Bone quality typically entails the following constituents; bone mineral density (BMD), bone microarchitecture, bone cell distribution (i.e. osteocytes, osteoblasts and osteoclasts), the distribution of microcracks or microdamage and the quality of the underlying organic bone matrix. Using both SR $\mu \mathrm{CT}$ and $\mu \mathrm{CT}$, bone density and microarchitecture are readily accessible; whereas, the other parameters have not been investigated to the same extent. However, they play an important role for bone stability and remodeling and have to be included into bone failure analyses. Microcracks caused by daily exercise are present even in healthy bone (17). As long as the density and magnitude of such cracks stays under a certain threshold, there is no significant change in bone strength; however, if the microcrack density and magnitude reach a certain threshold, there is a high probability for global failure (18). Thus, it is clearly beneficial to assess further bone quality parameters by $\mu \mathrm{CT}$. Due to their size, microcrack and cell density are parameters only detectable by SR $\mu$ CT. Even the spatial resolution of the current SR $\mu \mathrm{CT}$-systems does not seem to cover the numerous aspects of bone quality.

Although different studies have shown that including micro- and ultrastructural bone properties in the analysis of bone strength fracture risk on the statistical basis is beneficial, no mechanistic or constitutive modeling has emerged so far. One of the reasons is the limitation in observing actual bone failure modes. Consequently, image guided failure assessment (IGFA) combining mechanical testing and micro-computed tomography was developed (19-21). With this method it is possible to investigate bone failure in a time-resolved fashion where the actual "3D movie" of the failure process can be retrieved, step by step. IGFA has been only used in combination with $\mu$ CT so far. SR $\mu C T$ is believed to enhance insight into the failure process at the micro and ultrastructural level. The extraction of bone quality parameters (microcrack and cell density) allows investigating microcrack initiation and evolution in 3D. The possibility to measure the cell activity in bone allows gaining insights into remodeling of healthy and diseased bone; however, no stains have been adapted or developed for cell imaging using SR $\mu \mathrm{CT}$. This is especially true for osteoclasts and microcracks in bone, since the investigations were limited to 2D techniques such as LM and EM using fluorescent chelating agents (22) or heavy metal stains, respectively (23).

The work presented in this review is based on the general idea to provide tools for qualitative and quantitative characterization of hard and soft tissues in 3D using synchrotron light. These tools include the visualization and quantitative analysis of cell cultures in hydrated 3D environments using the absorption contrast mode of SR $\mu \mathrm{CT}$, the visualization and quantification of microcracks in bone to study bone failure, and the presentation of a device for image-guided failure assessment at the synchrotron radiation source. Results from the cell culture model system are presented and compared to classical cell imaging. From this model system, cells and scaffold can be segmented. It is shown that different cell phenotypes (fibroblasts and osteoblast-like cells) form distinct cell culture morphologies. The device for IGFA is validated, comparing the results to conventional mechanical testing. The uncovered features, microcracks and microfractures, are discussed in the context with the 2D tools conventionally used for the classification and quantification of microdamage. A preliminary algorithm for microcrack quantification is introduced. Finally, the 
comparison between the failure of fatigued and non-fatigued trabecular bovine bone shows a higher increase in the accumulated microcrack and microfracture volume in fatigued bone.

\section{MATERIALS AND METHODS}

\section{Cell Culture Preparation}

The cell cultures consist of human foreskin fibroblasts (HFF), rat tendon fibroblasts (3T3) or mouse calvaria osteoblastlike cells (MC3T3) seeded on poly-ethylene terephthalate (PET) multifilament yarns or a woven PET-textile. The yarns are made of 32 filaments with a rounded polygonal shape and an outer diameter of about $20 \mu \mathrm{m}$. The woven PET textile has a mesh size of $190 \mu \mathrm{m}$ and a filament diameter of $43 \mu \mathrm{m}$ The exact pre-treatment of the textiles, as well as the cell seeding procedures are described elsewhere (24). The seeded textiles were put onto a 3D shaker installed in a cell incubator. After 3 days of incubation, the yarn samples were removed from the incubator and fixed using $2.5 \%$ glutaraldehyde in phosphate buffered saline (PBS) (pH 7.4) at $4{ }^{\circ} \mathrm{C}$ for $5 \mathrm{~h}$. Subsequently, all except two of the yarn samples were stained with gold-labeled lectin (lectin from Triticum Vulgaris, Sigma Product number L1894) in tris buffered saline (TBS) ( $\mathrm{pH} \mathrm{7.4)} \mathrm{for} 3 \mathrm{~h}$ at a concentration of 1:10. One sample was stained, applying the same lectin at same concentration but for $6 \mathrm{~h}$, the other one was left unstained. The gold stain was amplified using a gold enhancement kit (Nanoprobes, Goldenhance - Lm/Blot). Prior to the amplification, all samples were washed twice in ultra-pure water for $10 \mathrm{~min}$. $200 \mu \mathrm{l}$ of the gold enhancement solution was added for $5 \mathrm{~min}$ with the exception of the one sample stained for $6 \mathrm{~h}$ with the lectin which was incubated with the gold enhancement for 20 min. Following amplification, the samples were washed in ultra-pure water once and in PBS twice. The samples were stored in PBS at $4{ }^{\circ} \mathrm{C}$ until tomographic imaging. For SR $\mu \mathrm{CT}$ measurements the yarns were inserted in a thin-walled glass vial filled with PBS. The vial was glued into a tomography sample holder using epoxy resin to seal the open end.

The woven textile sample was incubated with the $3 \mathrm{~T} 3$ cells until good confluence and then subjected to an intermittent cyclical loading regime composed of a $1 \mathrm{~h}$ stimulation phase at a $2 \%$ strain level with a frequency of $0.5 \mathrm{~Hz}$ followed by a $6 \mathrm{~h}$ resting phase. After 12 cycles, the mesh was taken out immediately following stimulation phase and fixed in $3 \%$ glutaraldehyde for 2 days in PBS at $4{ }^{\circ} \mathrm{C}$, stained with $\mathrm{OsO}_{4}$ for $1 \mathrm{~h}$ and subsequently washed with PBS once. The water in the sample was exchanged with ethanol (ETOH). For tomographic imaging, the sample was put into an Eppendorf tube filled with ETOH, which was glued onto the sample holder.

\section{Bone Sample Preparation}

Trabecular bovine bone cylinders were produced for mechanical testing and IGFA. Slabs were cut from a distal bovine femur using a band saw (FK23, Bizerba GmbH \& Co. KG, Balingen, Germany), the slab surface was perpendicular to the principal loading axis. Using a diamond trephine (MedArtis AG, Munich, Germany) and a drill press (Promac 212, Tooltek Co. LTD., Taichung Hsien, R.O.C.), cylindrical bone specimens of $3.7 \mathrm{~mm}$ diameter were cored from the slabs. The cores were cut to $4 \mathrm{~mm}$ long samples with a precision blade saw (Isomet 5000, Bühler LTD., Lake Bluff, Michigan, USA). Throughout the preparation procedure, the bone samples were constantly irrigated with phosphate buffered saline (PBS) ( $\mathrm{pH}$ 7.4) to cool them and preserve the bone properties during the production process. Bone marrow was extracted from the cylinders using a $0.2 \%$ handsoap (Johnson Diversey, Sturtevant, USA) in purified water and an ultrasonic bath. The samples immersed in the handsoap solution were placed in ultrasonic bath for a period of 90 min, keeping the water temperature constantly below $40{ }^{\circ} \mathrm{C}$. The solution was exchanged and the samples were rinsed with ultra-pure water. This process was repeated up to 5 times until no more bone marrow was visible under a light microscope. After marrow extraction, the samples were kept in PBS at $4{ }^{\circ} \mathrm{C}$. For mechanical testing and IGFA, the samples were glued onto $1 \mathrm{~mm}$ thick brass plates, $4 \mathrm{~mm}$ in diameter using cyano-acrylate super-glue (Loctite 431, Henkel KgaA, Düsseldorf, Germany). The samples were exposed to air until the glue was dry and placed into PBS for re-hydration. Bone samples where subjected to mechanical testing within the next $6 \mathrm{~h}$. For IGFA in combination with SR $\mu \mathrm{CT}$ imaging, two similar samples were selected by scanning them with a desktop tomography system ( $\mu \mathrm{CT} 40$, Scanco Medical AG, Bassersdorf, Switzerland). The selection was based on similar sample morphology, i.e. the values for relative bone volume (BV/TV), bone surface (BS) and structure model index (SMI) were used.

Table 1: Morphological parameters used to select two similar samples used for IGFA.

\begin{tabular}{|c||c|c|c|}
\hline Sample / Description & BV/TV [\%] & BS $\left[\mathrm{mm}^{2}\right]$ & SMI \\
\hline Sample A (not fatigued) & 23.45 & 140.30 & 0.77 \\
\hline Sample B (fatigued) & 22.99 & 141.29 & 0.68 \\
\hline
\end{tabular}


$\mathrm{BV} / \mathrm{TV}$ indicates the total amount of bone within the cylinder, the BS is a measure for the number of individual trabeculae and the SMI gives their mean shape, it is a parameter indicating whether the observed trabeculae are rather rod or plate-like (25). The values for the selected samples are given in Table 1. The SMI of 0.7-0.8 indicates that both samples are a hybrid case between plates and rods, being closer to the plate-like shape. The two selected samples were stored in PBS soaked gauze at $-20{ }^{\circ} \mathrm{C}$ until the IGFA experiment was performed. Then the samples were thawed immersing them in PBS kept at room temperature.

\section{Mechanical Testing of Bone and Aluminum}

For validation, the mechanical tests on engineered and biological cylindrical specimens made from solid aluminum and trabecular bovine bone were performed using the in situ mechanical loading and staining device (IMCSD) and a commercial testing device (Model 1456, Zwick GmbH \& Co. KG, Ulm, Germany). The IMCSD was specifically developed to perform image guided failure assessment experiments at the materials science (MS) beamline of the Swiss Light Source (SLS). 3D design drawings of the IMCSD are shown in the results part. The device was designed to house cylindrical bone samples of $4 \mathrm{~mm}$ in height and $3.7 \mathrm{~mm}$ in diameter. The IMCSD consists of three major parts: The sample chamber, consisting of brass and a radiolucent plastic tube, made of Torlon 4203 (Solvay S.A., Brussels, Belgium) a poly-amid-imide is represented in the lower part. It is directly screwed onto the tomography sample handler. The actuator housing and mechanical guides are in the upper part and connected via a bearing. Once the sample chamber is screwed to the actuator housing it can both rotate as a unit about the fixed guides and be driven by the rotation axis of the sample handler. The displacement-exerting device is a piezoelectric actuator (PI P-246K020, Physics Instruments $\mathrm{GmbH}$, Karlsruhe, Germany). It is capable of exerting $300 \mu \mathrm{m}$ displacement, with the accuracy of $0.1 \mu \mathrm{m}$. The temporal stability of exerted displacement is guaranteed by the internal closed loop system of the actuator. The actuator can be used to apply static and dynamic loads on the specimen kept in the sample chamber. The force sensor is directly put on top of the actuator pin. Two load cells are currently available for the device with ranges from $0 \mathrm{~N}$ to $100 \mathrm{~N}$ (Burster 8413-100, Burster Präzisionsmesstechnik GmbH \& Co. KG, Gernsbach, Germany) and $500 \mathrm{~N}$ (Burster 8413-500, Burster Präzisionsmesstechnik GmbH \& Co. KG, Gernsbach, Germany) and an accuracy of $0.5 \mathrm{~N}$ and $2.5 \mathrm{~N}$ respectively. The displacement of the load cells due to their own compliance was measured in calibration experiments. The acquired displacement data are corrected using the calibration. The piezoelectric actuator is controlled using a high voltage amplifier (Physics Instruments GmbH, Karlsruhe, Germany) connected to the PC via a RS-232 serial interface. The load cell is read out using a MVD255 signal amplifier (Hottinger Baldwin Messtechnik GmbH, Darmstadt, Germany), which is connected to the PC via a RS-485 interface through a NI PCI-485/4 PC card (National Instruments, Austin, Texas, USA). The whole ICMSD setup is controlled with a PC using LabView (National Instruments, Austin, Texas, USA). The valves attached to the sample chamber are for applying physiologic fluid to the sample. The solid aluminum cylinders used for mechanical tests were $4 \mathrm{~mm}$ in height and $3.7 \mathrm{~mm}$ in diameter (alloy Anticorodal 112, Häuselmann Metall, Dietikon, Switzerland). Aluminum samples were weighed and divided into two similar groups of five samples each. Continuous mechanical tests were then performed using the same testing parameters for both devices. The aluminum cylinders were subjected to a preload of $10 \mathrm{~N}$ then continuous testing was performed applying a strain rate of $0.12 \% / \mathrm{s}$. Samples were loaded up to $500 \mathrm{~N}$ being the limit for the IMCSD. The trabecular bone samples were divided randomly in two groups of five samples each. Mechanical testing was performed as follows. A preload of $2 \mathrm{~N}$ was applied then continuous mechanical tests with a strain rate of $0.12 \% / \mathrm{s}$ were performed. Bone samples were compressed up to a maximum of $7 \%$ strain, i.e. until the maximum displacement of $300 \mu \mathrm{m}$ for the IMCSD was reached.

\section{Synchrotron Radiation Micro-Computed Tomography}

Tomography experiments were either performed at the MS beamline (26) at the SLS of the Paul Scherrer Institute (PSI) or in the case of the PET mesh at beamline BW 2 (27) of the Hamburger Synchrotronstrahlungslabor (HASYLAB) at the Deutsches Elektronensynchrotron (DESY). For each cell-yarn sample imaged at the MS beamline of the SLS 1000 radiographic projections were recorded at the photon energy of $14.5 \mathrm{keV}$. The chosen energy gives the highest contrast for the applied gold stain in absorption contrast mode $(24,28)$. The nominal spatial resolution, i.e. pixel width, of the acquired projections was $0.7 \mu \mathrm{m}$. A distance of about $50 \mathrm{~mm}$ was allowed between sample and detector in order to achieve edge enhancement effect and contrast of the yarn. In the case of the PET mesh, 720 radiographic projections were recorded at the photon energy of $24 \mathrm{keV}$ and at a spatial resolution of $3.5 \mu \mathrm{m}$, which was determined using a gold edge (29). Prior to tomographic reconstruction using a filtered back-projection algorithm, all raw data were binned by a factor of two in order to improve the signal to noise ratio in reconstructed slices (30). Thus the nominal resolution in reconstructed slices was $1.4 \mu \mathrm{m}$ and $4.7 \mu \mathrm{m}$ for the yarns and the woven textile, respectively. All IGFA experiments were performed at the MS beamline of the SLS at the photon energy of $20 \mathrm{keV}$. The pixel size in each of the recorded 
projections was $3.5 \mu \mathrm{m}$. These data were subsequently binned by a factor of two prior to the reconstruction. Each sample had to be scanned 3 times at different heights at every compression step recorded during IGFA, since the used X-ray beam was less than $2 \mathrm{~mm}$ in height. The used tomography setup had a field of view (FOV) of $7 \mathrm{~mm}$ by $7 \mathrm{~mm}$. Thus, the tube made of Torlon around the sample with an outer diameter of $10 \mathrm{~mm}$ was not entirely in the FOV of the detector, i.e. local tomography was performed. This resulted in an offset of reconstructed absorption values and in a typical bright ring shaped artifact or two half ring shaped artifacts within reconstructed slices (31).

\section{Scanning Electron Microscopy}

Two samples were imaged with scanning electron microscopy (SEM) using the S-900 SEM (Hitachi HighTechnologies Ltd., Japan), one was the woven PET textile seeded with 3T3 cells, prior imaged using SR $\mu$ CT. The other one was the PET yarn seeded with HFF cells and stained with the gold-labeled lectin and gold enhancement. Both samples were dehydrated and critical point dried. The PET mesh was sputtered with a $4 \mathrm{~nm}$ thin film of silver, whereas the yarn was sputtered with a $5 \mathrm{~nm}$ thin film of chromium. Both samples were imaged at the acceleration voltage of $15 \mathrm{keV}$ using the secondary electron (SE) signal in the case of the woven PET textile and the backscattered electron (BSE) signal in the case of the yarn.

\section{Image Guided Failure Assessment}

After insertion into the IMCSD sample chamber, where the specimens were kept immersed in PBS, a preload of $5 \mathrm{~N}$ was applied, and the samples were preconditioned by compressing them seven times to $0.3 \%$ strain. For sample B the preload was considerably higher, namely $10 \mathrm{~N}$, since the specimen glued into the brass endcaps was slightly longer than accepted by the experimental setup. Prior to any mechanical testing the radiographic images were used to measure the exact sample height, resulting in $4071.5 \mu \mathrm{m}$ for sample A and $4175.4 \mu \mathrm{m}$ for sample B. After the preconditioning, the elastic modulus was determined by compressing the specimen up to $0.3 \%$. Sample B was additionally fatigued applying 3500 displacement cycles between $0 \%$ and $0.5 \%$ strain. After each $500^{\text {th }}$ cycle the dynamic compression was stopped and a hysteresis curve was recorded compressing the sample to $0.5 \%$ strain. Fatigue was defined as a $10 \%$ reduction in modulus. For IGFA both samples were tomographically scanned at $0 \%, 1 \%$ and $2 \%$ strain. The fatigued sample without strain was scanned twice, before and after the application of the dynamic displacement cycles. During all IGFA experiments the applied displacements were crosschecked with values derived from radiographic projections.

\section{Data Processing}

The data preprocessing of SR $\mu \mathrm{CT}$ images of the yarn samples was performed similarly to the one described in another communication (32): In order to reduce the amount of data a cylindrical region of interest (ROI) was selected for each dataset retrieved from SR $\mu$ CT containing only the cell-seeded yarn in PBS. The resulting histograms of all measurements were fitted with two Gaussians, one for the stained cells and one for the PBS and the yarn, using the Levenberg-Marquardt algorithm (ProFit, QuantumSoft, Uetikon am See, Switzerland). From these fits the two distinct thresholds were retrieved for the two datasets, omitting most of the background (PBS) voxels. After thresholding the data were subjected to a component labeling (33) procedure eliminating all components with a volume smaller than 3/4 of the estimated cell volume of $4189 \mu \mathrm{m}^{3}$ (approximating a sphere with $10 \mu \mathrm{m}$ radius). After this step the stained cells and the yarn filaments in the original dataset were retrieved. In the case of the woven textile the dataset was smoothed applying a 3D Gaussian filter (sigma 1.2 voxels on a $3 \times 3 \times 3$ voxel base). The resulting histogram of the dataset is distorted. The reason is that the sample did not fully fit into the field of view (FOV) of the detector during the measurement. The lower end of the conical shaped Eppendorf tube was inside the FOV, whereas the higher parts of the container were outside the FOV. Due to this partly local tomography scan, the different slices had different absolute local absorption. Therefore, first a cylindrical ROI was set to exclude the Eppendorf container in order to reduce the amount of data. Subsequently the ROI was further cropped to an even smaller region. A threshold varying with each slice of the dataset was be computed by analyzing 3 different slices, at the top, bottom and middle slice. The threshold was interpolated in a linear fashion between the top and middle slice and held constant between the middle and bottom slice. After applying the thresholds the resulting mask was subjected to 3D component labeling eliminating all but the largest particle. Subsequently, the mask was enlarged applying once the morphological dilation operation (34), to overcome the partial volume effect. With this final ROI the initial dataset was masked.

The datasets retrieved from the bone samples at the different heights were joined to a single dataset at each compression step. Then a cylindrical ROI was chosen in order to exclude the artifacts arising from the local tomography performed. Subsequently the dataset was smoothed applying a 3D Gaussian filter (sigma 1.2 voxels on a $3 \times 3 \times 3$ voxel base) and thresholded using an automated adaptive thresholding algorithm. For visualization the binarized data were segmented in endplates and bone in a semi-automatic manner, and the binarized datasets were all subjected to a component-labeling algorithm in order to remove all but the largest component. Subsequently the datasets were aligned at the lower, notmoving endplate using a cross-correlation routine. 


\section{RESULTS}

Figure 1 shows the reconstructed slices of unstained and lectin stained HFF cells on a PET multifilament yarn. It is easy to recognize that the lectin stain combined with the gold enhancement is effective to uncover the cells, which appear bright. The stained cell culture encapsulates the yarn. A similar shaped feature, very weak in contrast, is detected in the reconstructed slice of the unstained sample. Here the edge enhancement, originating from the highly coherent synchrotron radiation, uncovers not only the yarn filaments but also the surface of the cell culture (white arrows in Figure 1). The edge enhancement alone, however, is insufficient for cell culture visualization. This means that besides the absorption contrast applied in combination with a contrast agent, previously validated qualitatively (24) and quantitatively (32), quantitative phase contrast (35) is a potential technique to image the cell culture without any stain. The streaks seen in the reconstructed slice of the unstained sample shown in Figure 1 on the left are from the result of sample movement and slightly improper selection of the rotation center for the tomographic reconstruction. Nonetheless the slice indicates the possibility to apply quantitative phase contrast tomography for cell culture visualization. The fact that besides the whole cell culture also single cells can be detected is represented in Figure 2 . Here, a radiographic projection of a sample stained with the gold labeled-lectin and the gold enhancement is compared to the SEM micrograph of a comparable sample. The magnified detail of the radiographic projection and the SEM micrograph have identical scale. The image width is $118 \mu \mathrm{m}$. Since the backscattered electrons are recorded for the SEM image bright spots are associated with higher atomic numbers, i.e. the applied gold stain. The observed feature in the magnified detail of the projection resembles the SEM micrograph, showing a single cell sitting on a yarn filament. A part of the entire culture of 3T3 cells on the woven PET textile is shown in Figure 2, too. The 3D visualization of the reconstructed dataset is compared to the SEM micrograph of the same sample. The similarities are clearly seen, despite the fact that the visualization from SR $\mu \mathrm{CT}$ is of lower spatial resolution. It is based on a 3D model of the sample and not only the surface image of the sample as retrieved by SEM. Already after binarization of the dataset it is possible to extract quantitative values characterizing the cell culture. So far the efforts were based on tomograms acquired at synchrotron radiation sources of second generation with very weak edge enhancement and thus it was not possible to retrieve information of the scaffold geometry (32). This problem is mastered by synchrotron radiation produced by facilities of third generation. Due to the higher coherence the edge enhancement is clearly seen in reconstructed images (cp. Figure 1) for both the cells and the filament surfaces. In terms of data processing the situation becomes difficult, since the yarn and the cell culture cannot be segmented from each other by simple thresholding. For this purpose a semi-automatic algorithm has been developed, based on component labeling and morphological dilation operation. Applying the knowledge that the scaffold consists of 32 filaments the entire dataset was analyzed slice by slice in the three directions (sagittal, frontal and axial). Each slice was inverted and subjected to 2D component labeling omitting the largest component. This was repeated several times. Finally, the guess for the hollow insides of the filaments was refined manually. Subsequently, the morphological dilation operation was applied to enlarge the scaffold mask until the mean thickness reached $20 \mu \mathrm{m}$. Then the mask was applied to the thresholded dataset.

No Stain

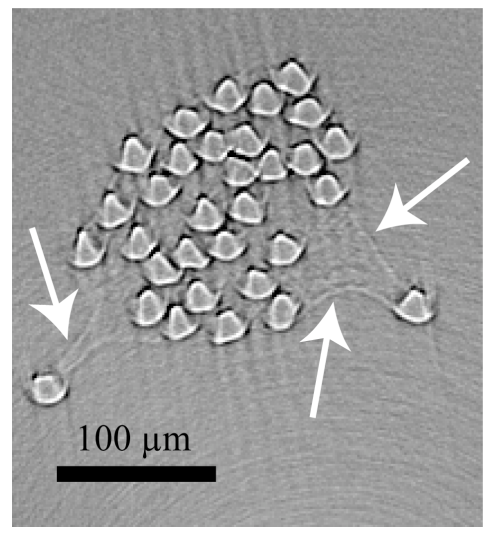

Lectin Stain

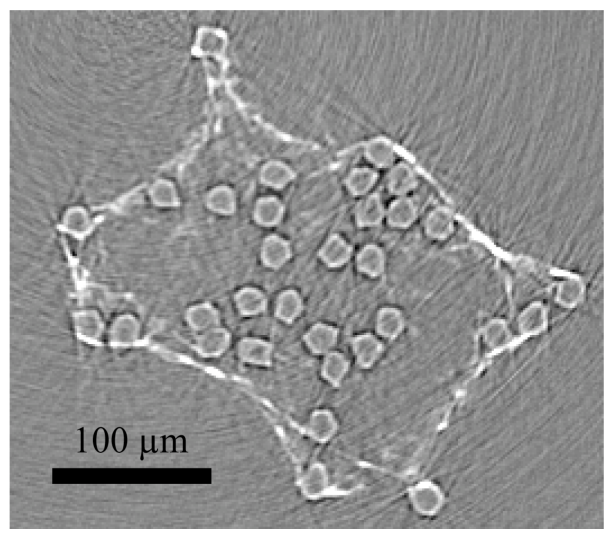

Figure 1: HFF cells on a PET multifilament yarn unstained and stained using gold-labeled lectin and gold enhancement as indicated. The data were acquired at the MS beamline (SLS), photon energy $14.5 \mathrm{keV}$, voxel length $1.4 \mu \mathrm{m}$ after 2 -fold binning of the raw data. 

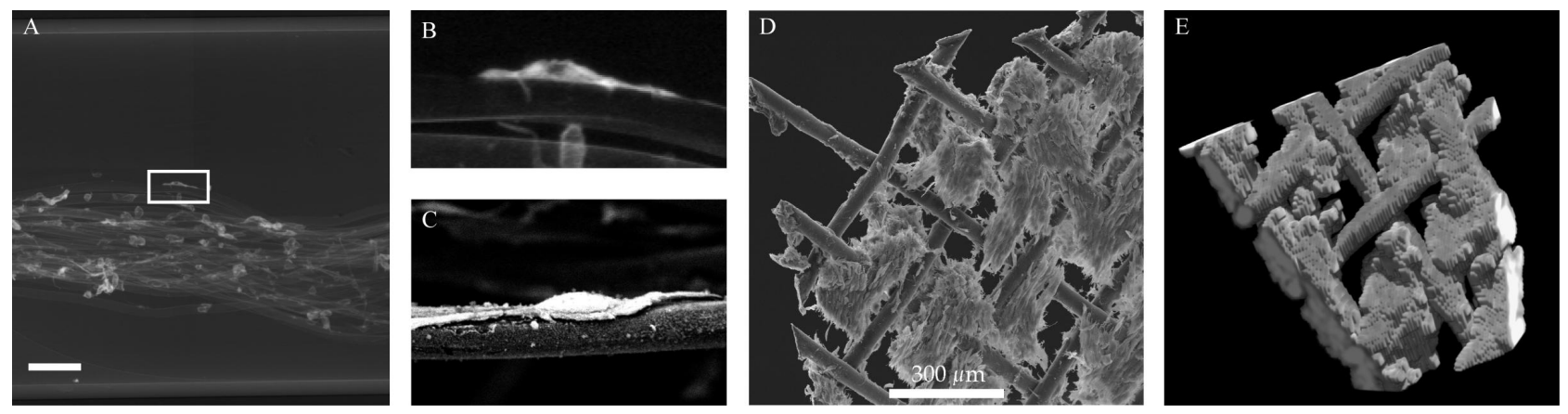

Figure 2: A: Projection of an HFF cell-seeded yarn, stained with lectin and gold enhancement. Image data acquired at ESRF, beamline ID 22, photon energy $15 \mathrm{keV}$, voxel length $0.66 \mu \mathrm{m}$, scale bar $100 \mu \mathrm{m}$ B: Region of interest from A (white box), indicating a single HFF cell, image width corresponds to $118 \mu \mathrm{m}$. C: SEM image (backscattered electrons) of a comparably stained sample illustrating the feature shown in B. Image width $118 \mu \mathrm{m}$, electron acceleration voltage $15 \mathrm{kV}$. D: SEM micrograph of a dehydrated sample consisting 3T3 fibroblasts seeded on PET fabric, stained with $\mathrm{OsO}_{4}$. Mesh size $190 \mu \mathrm{m}$, filament size $43 \mu \mathrm{m}$. Backscattered electrons, primary electron energy $15 \mathrm{keV}$. E: Section of the tomogram of the same hydrated sample taken at HASYLAB BW 2 at a photon energy of $24 \mathrm{keV}$ and a spatial resolution of $3.5 \mu \mathrm{m}$.

The retrieved cell culture was subsequently subjected to component labeling excluding all particles below $3 / 4$ of the assumed cell size (equal to a sphere with $10 \mu \mathrm{m}$ diameter). Additionally the cell volume representing the MC3T3 cells was cleaned manually from artifacts resulting from local absorption maxima. The algorithm is described in further detail elsewhere (36). This procedure was performed on the datasets retrieved from two samples, both cell seeded yarns but with different cell phenotypes, i.e. HFF or MC3T3 cells. The qualitative outcome of these two experiments is given in Figure 3. Here 3D visualizations of the cells together with the scaffold are presented well along with 3D visualizations of the adhesive surfaces of the cell cultures on the yarn scaffold. Due to the segmentation described above it is possible to quantify the cell-culture yarn system. Similar to previously published work (32) the quantification was done slice-by-slice, with the slices being aligned perpendicularly to the long yarn axis. The total yarn volume in each slice was determined by triangulating all filament points and projecting the retrieved triangles onto the image grid. Subsequently quantity and radial distribution of total yarn volume (TV), cell volume (CV) and filament volume (FV) were computed. Summing the information obtained from all slices the overall values of the parameters were computed. Additionally, values for mean cross-sectional area, mean envelope radius, relative cell volume $(\mathrm{CV} / \mathrm{TV})$, relative filament volume (FV/TV), and cell density are obtained. The mean envelope radius was computed from the mean cross-sectional area assuming a circular shape. Using a marching cubes algorithm (37) values for cell culture surface (CS), (to the yarn) adhesive cell culture surface (aCS) and cell volume (CVmc) were computed. An overview of the derived parameters is given in Table 2. It can be seen that yarn geometries are fully comparable, and $\mathrm{CV} / \mathrm{TV}$ is identical with $10 \%$ and $11 \%$ for the HFF cells and the MC3T3 cells, respectively.

Table 2: Quantified parameters of the investigated cell cultures, standard deviations are given where applicable.

\begin{tabular}{|l|cc|}
\hline \multicolumn{1}{|c|}{ Sample } & HFF & MC3T3 \\
\hline \hline Total yarn volume $\left[\mathrm{mm}^{3}\right]$ & 0.063 & 0.086 \\
Total cell volume $\left[\mathrm{mm}^{3}\right]$ & 0.006 & 0.009 \\
Mean cross sectional area $\left[\mathrm{mm}^{2}\right]$ & $0.064 \pm 0.004$ & $0.082 \pm 0.007$ \\
Mean envelope radius $[\mu \mathrm{m}]$ & $143 \pm 9$ & $162 \pm 15$ \\
CV/TV [\%] & $10 \pm 1$ & $11 \pm 2$ \\
FV/TV [\%] & $14 \pm 1$ & $11 \pm 1$ \\
Cell density $\left[\mathrm{mm}^{-3}\right]$ & 24000 & 26000 \\
aCS/CV $\left[\mathrm{mm}^{-1}\right]$ & 3.8 & 69.8 \\
aCS/CS $[\%]$ & 0.7 & 14.5 \\
\hline
\end{tabular}


HFF
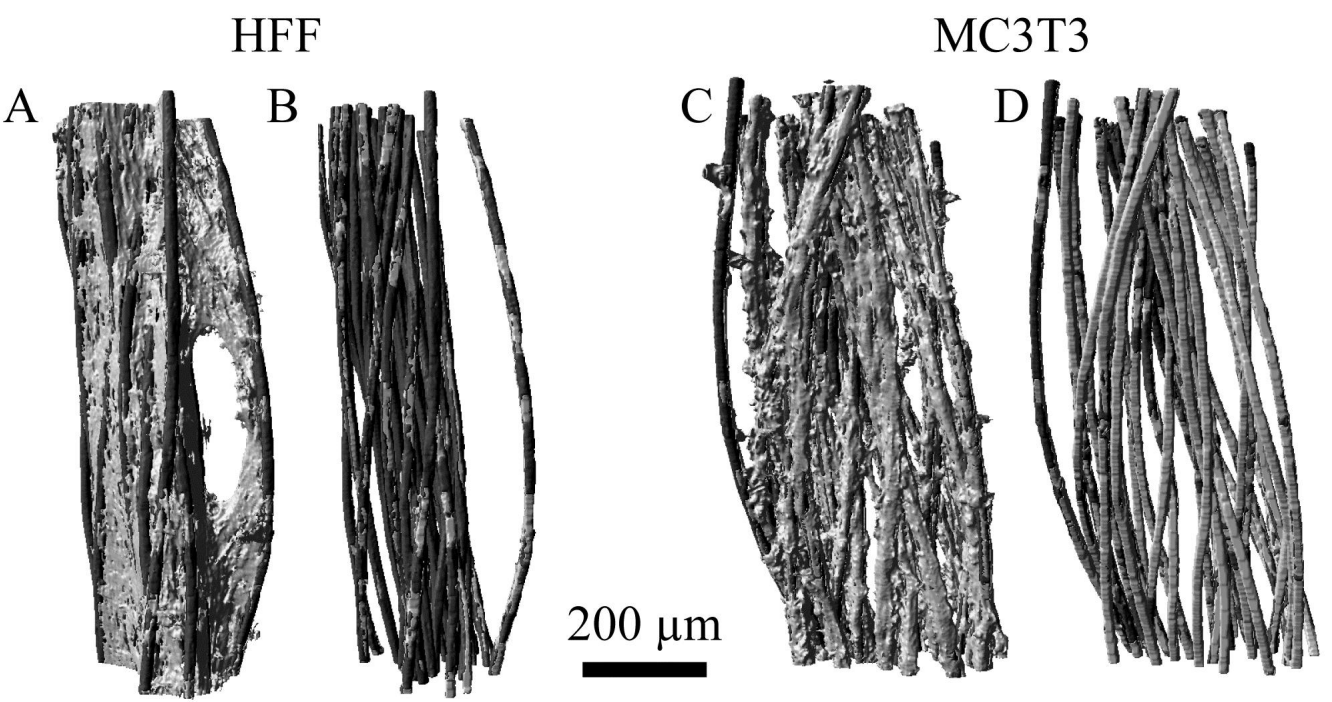

Figure 3: 3D visualization of the cell cultures $(A, C)$ and their adhesive surfaces $(B, D)$ (both light gray) on the yarn (dark gray). The HFF cells build a tent-like structure, whereas the MC3T3 cells are lining the individual filaments. The adhesion surface of HFF cells seems to be greatly reduced compared to the MC3T3 cells. Data acquired at the MS beamline (SLS), photon energy $14.5 \mathrm{keV}$, voxel size $1.4 \mu \mathrm{m}$.

The same applies for the cell densities, as the cell volume of $4189 \mu \mathrm{m}^{3}$ was assumed in both cases. The only remarkable differences are the ratios of adhesive cell culture surface to total cell culture volume $(\mathrm{aCS} / \mathrm{CVmc})$ and total cell culture volume $(\mathrm{aCS} / \mathrm{CS})$. Here, the values differ by one to two orders of magnitude, showing a difference in the in-growth pattern of the two cell types. This finding is confirmed by the 3D representations of the datasets shown in Figure 3. The HFF cells form a tent-like structure around the yarn, whereas the MC3T3 cells are lining the yarn filaments. Furthermore the 3D visualizations of the adhesive cell surface in Figure 3 underline the large differences computed for $\mathrm{aCS} / \mathrm{CS}$ and $\mathrm{aCS} / \mathrm{CV}$. From Figure 3 it seems that the HFF cells are rather inhomogeneously distributed within the yarn in contrast to the MC3T3 cells. This inhomogeneity of the cell distribution of the HFFs is also demonstrated in Figure 4, where CV is plotted versus distance to the closest filament. This plot was generated by iterative dilation of the segmented filaments and masking of the segmented cells underneath. As the MC3T3 cells can be assumed to be homogeneously distributed, the HFF cells show a much lower peak close to the filaments and are much more broadly distributed. In contrast, already $50 \%$ of the MC3T3 volume is within a distance of $2.8 \mu \mathrm{m}$ to a filament surface.

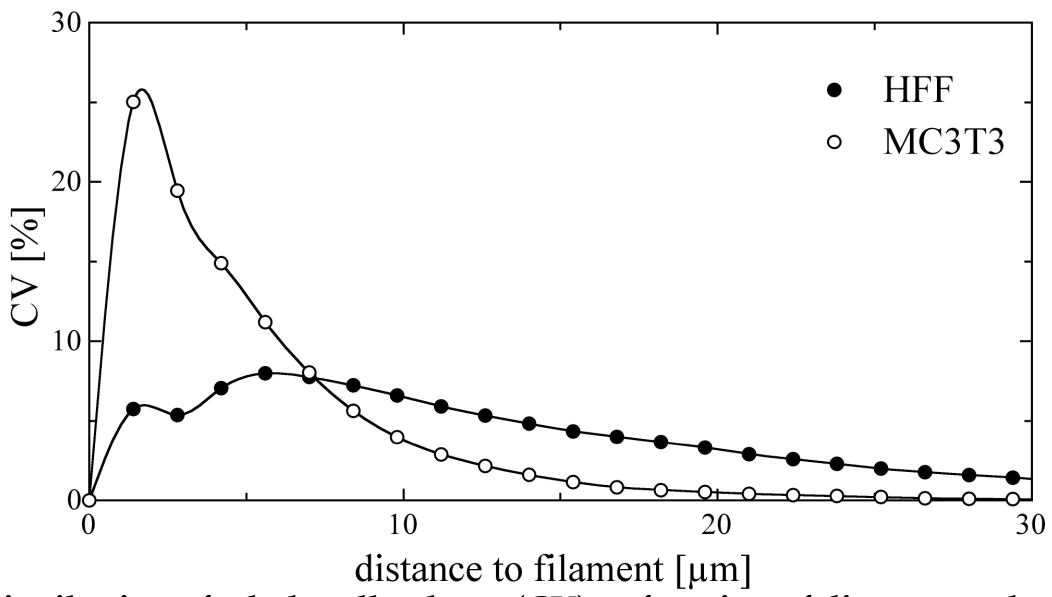

Figure 4: Distance distribution of whole cell volume $(\mathrm{CV})$ as function of distance to the closest filament surface. $59 \%$ of the MC3T3 cells are located within $4.2 \mu \mathrm{m}$ to the closest filament surface, compared to $18 \%$ of the HFF cells. The double peak in the HFF cell distribution is due to the tent-like morphology. 

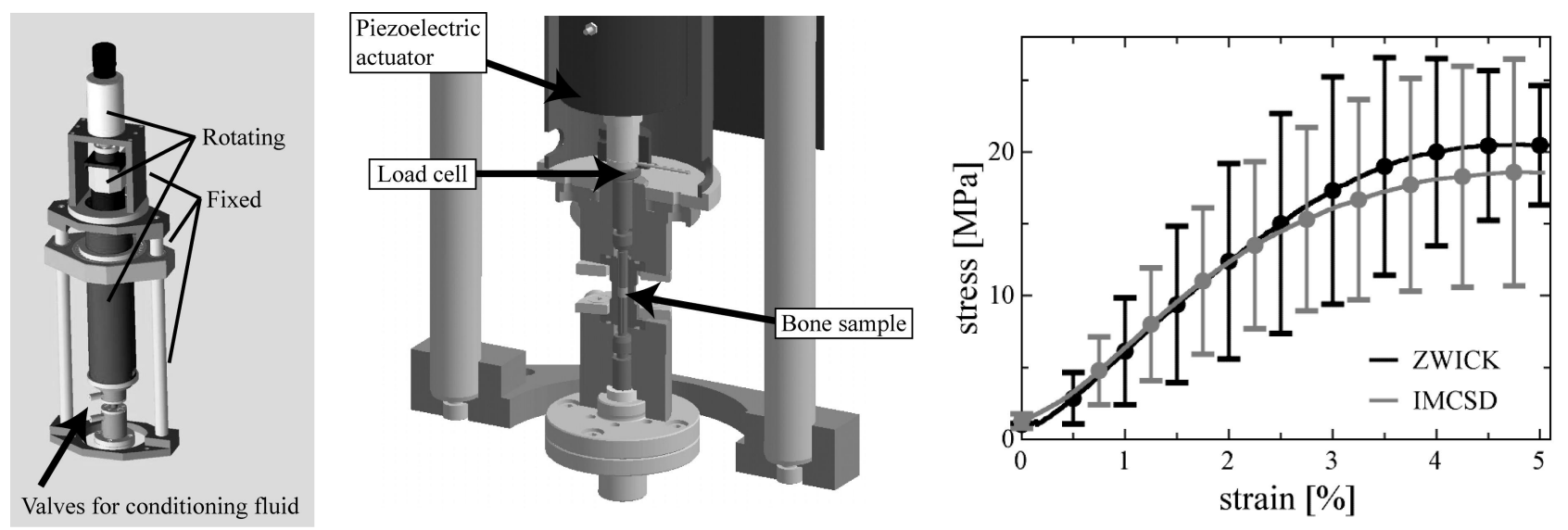

Figure 5: 3D design drawings of the whole IMCSD device (left), and the sample chamber (middle), which is screwed to the actuator housing and the tomography rotation stage. Average corrected stress strain curves (right) of the trabecular bone specimens tested using the conventional mechanical testing device and the IMCSD. The error bars retrieved from the IMCSD are shifted by $0.25 \%$ strain for better visibility.

In Figure 5 the IMSCD design is illustrated. The device was designed and built to apply static and dynamic compression on bone samples, while performing tomographic imaging. The results of the mechanical tests with the aluminum samples were combined in the form of an average curve for both devices used (not shown). In order to calculate the average curves the individual stress-strain curves were re-sampled onto a common strain grid with a pitch of $0.01 \%$. Since the IMCSD showed to have a considerable compliance the data acquired were corrected using the average curve of the aluminum specimens retrieved from the conventional mechanical testing system. The compliance of the device was computed from these results to be $(0.13+/-0.02) \mu \mathrm{m} / \mathrm{N}$. This value was used to correct the displacement values measured with the IMCSD using the corresponding force data for the mechanical tests performed. The elastic modulus of the aluminum specimens was computed by a linear regression in the range between $1 \%$ and $2 \%$ strain of the average curve retrieved from the conventional mechanical testing system to be $(5.246 \pm 0.006) \mathrm{GPa}$. The average of the corrected curves retrieved from the mechanical testing of the trabecular bovine bone cylinders are given in Figure 5. The curves without correction (not shown) had similar shape, but were slightly shifted from each other, presumably due to varying toe regions. In fact one curve retrieved from the conventional mechanical testing system was excluded for the computation of the average curve, since it had a toe region extending to $3.5 \%$ strain. In order to correct for the toe regions overall the following procedure was applied. The elastic modulus was fit in the region between $1 \%$ and $2 \%$ strain, where all samples showed an elastic behavior. The regression curve from each modulus fit was then used to correct the strain values in the following manner. Zero strain was set at the intersection of the regression line with the strain axis (at zero stress). The corrected curves were then used to compute yield strain and ultimate strain. An overview of the mechanical parameters is given in Table 3. Figure 5 shows the average curves from the strain-corrected curves, which exhibit very similar shape.

Table 3: Quantified mechanical parameters of the trabecular bovine bone samples, from the conventional mechanical testing device and the IMCSD. The p-values from the t-test of the individual results demonstrate that there is no significant difference between the results.

\begin{tabular}{|l||lllllll|}
\hline Device & \multicolumn{3}{c|}{ ZWICK } & \multicolumn{3}{c|}{ IMCSD } & t-test \\
\hline Parameter & Average & Std Dev. & $\mathrm{N}$ & Average & Std. Dev. & $\mathrm{N}$ & p-value \\
\hline \hline Elastic Modulus [MPa] & 562.8 & 338.4 & 5 & 613.1 & 257.6 & 5 & 0.798 \\
Stiffness [N/um] & 1.51 & 0.91 & 5 & 1.65 & 0.69 & 5 & 0.798 \\
Yield Strength [MPa] & 21.09 & 5.32 & 3 & 16.79 & 6.15 & 5 & 0.357 \\
Yield Strain [\%] & 3.32 & 0.92 & 3 & 3.12 & 1.02 & 5 & 0.788 \\
Ultimate Compressive Strength [MPa] & 22.49 & 5.17 & 4 & 18.83 & 7.78 & 5 & 0.448 \\
Ultimate Compressive Strain [\%] & 5.37 & 1.41 & 4 & 4.76 & 0.86 & 5 & 0.452 \\
\hline
\end{tabular}




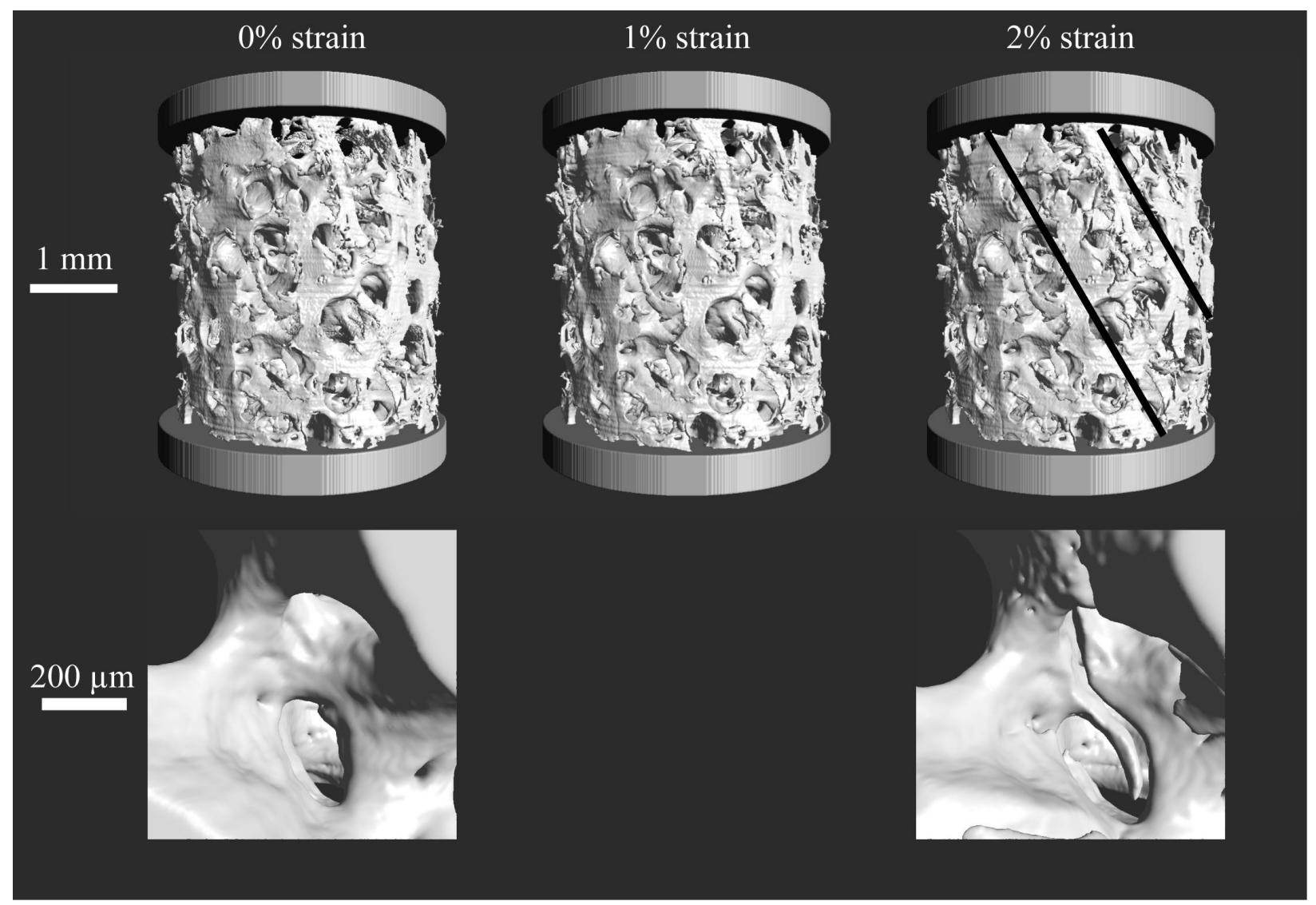

Figure 6: 3D visualization of a detail and the whole non-fatigued bone sample (sample A) subjected to IGFA at different compression steps. The sample shows a fracture band going diagonally from the upper left to the lower right through the sample (indicated with the black lines, the failure band is situated in between them). The detail shows the microcrack formation. Data acquired at the MS beamline (SLS) at the photon energy of $20 \mathrm{keV}$.

Only the average ultimate stress for the samples tested with the conventional testing device is higher but compared to the standard deviation of the average stress values well within the variation between the individual samples. The coefficient of variation (COV) for the parameters retrieved using the conventional testing machine ranged between $23 \%$ and $60 \%$, whereas the COV for the parameters retrieved using the IMCSD ranged between $18 \%$ and $42 \%$. A regression analysis of the average mechanical properties gave a high correlation with an $\mathrm{r}^{2}$ value of $1(\mathrm{p}<0.0001)$. All parameters quantified from the curves retrieved using the conventional mechanical testing device overlapped with the ones retrieved with the IMCSD with p-values always greater than 0.05 as presented in Table 3. Figure 6 shows 3D visualizations of a detail as well as the whole bone sample subjected to IGFA that was not fatigued. This sample exhibits a fracture band at $2 \%$ strain ranging through the whole sample from the top left to the bottom right, as indicated by the black lines in Figure 6. Especially the detail in the same Figure shows the power of IGFA in combination with $\mathrm{SR} \mu \mathrm{CT}$ : microscopic cracks, i.e. microfractures and microcracks are uncovered in a 3D manner. In order to quantify the observed microcracks and microfractures in bone an algorithm for their detection was developed based on the morphological dilation and erosion procedure. The algorithm was performed on the binarized bone structure. First the morphological dilation was performed for a few times. The exact number of repetitions is dependent on the maximum crack thickness, which shall be detected. This crack thickness can be estimated as follows: each dilation step closes a crack up from two sides, i.e. it detects cracks with a thickness of two voxel edge lengths. Subsequent to the dilation operations the morphological erosions were preformed. The number of erosion steps is the same number as dilation steps plus one, in the case presented here 7 dilation and 8 erosion steps. Finally the retrieved volume is used as a mask on the inverted bone volume data. Left after the masking procedure are the cracks, but also internal voids, and depending on the number of dilations performed also a considerable volume on the bone surface. 


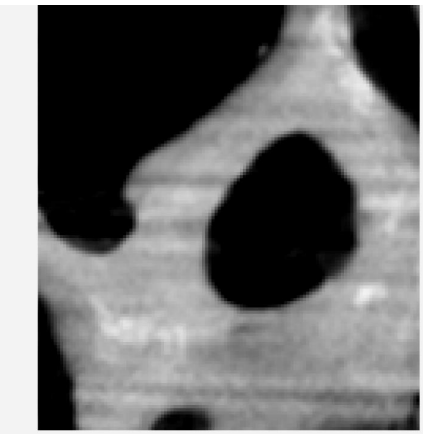

$200 \mu \mathrm{m}$
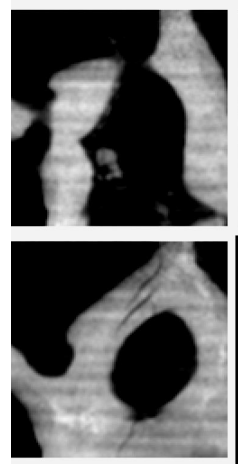

$200 \mu \mathrm{m}$
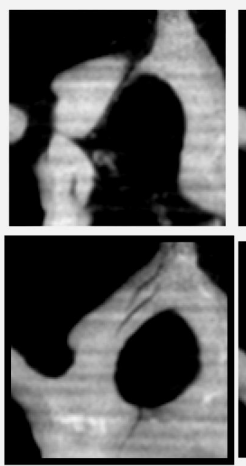

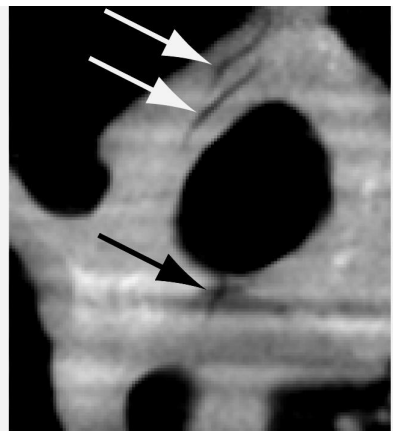

$1 \%$ strain
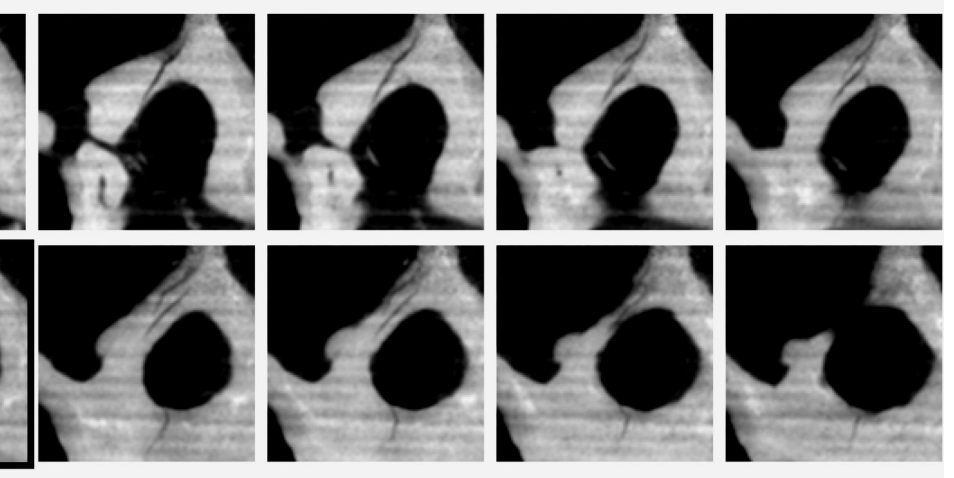

$2 \%$ strain

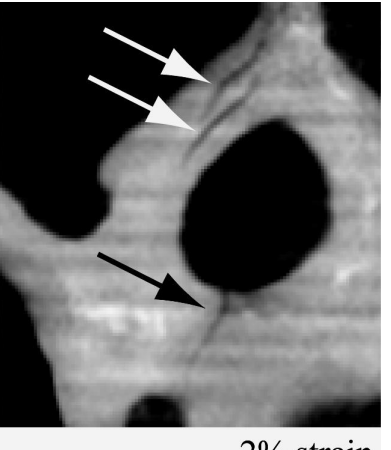

Figure 7: Detail of virtual, $7.5 \mu \mathrm{m}$ thick sections through sample $\mathrm{A}$ at the different compression steps (top). In the images two internal (white arrows) and one surface connected (black arrow) microcrack can be distinguished. The classification internal is actually misleading as shown in the image series presented at the bottom (corresponding slice to the one at top has a black frame). In fact the two internal cracks are one crack extending over a whole trabecula being connected to the bone surface, i.e. a microfracture. The horizontal streaks seen in the slices are due to features present in the incoming X-ray beam. Data were acquired at the MS beamline (SLS), photon energy of $20 \mathrm{keV}$.

The latter is selected due to the trabecular morphology. This means that the quantitatively detected crack volume is always higher than the actual crack volume. It is, however, still possible to compare the development of crack volume in a specimen subjected to a series of compression steps as done during IGFA using the retrieved crack volume at zero strain as the baseline. Besides the crack detection another important parameter of interest is, whether the microcracks are in connection with the bone surface or if they are purely internal. The reason for this is due to the classical 2D methods used to study microcracks, i.e. histology and microscopy. In such investigations microcracks are usually classified for quantitative analysis as either internal or surface connected cracks (38-40). In compact bone the number of surface connected cracks is small (41). Thus an algorithm was developed scanning the detected cracks, dividing them into internal and surface-connected cracks and computing their volume. Cracks that range through a whole trabecula are usually not considered as microcracks but are referred to as microfractures (42). The fact that the conventional classification of microcracks and microfractures from 2D images is ambiguous is proven in Figure 7. Here virtual 2D cuts through the sample are displayed: at the top a virtual cut through similar regions of the sample at zero, $1 \%$ and $2 \%$ strain is shown. Cracks indicated by the arrows are forming at $1 \%$ strain and are propagating increasing the strain to $2 \%$. The three cracks that can be distinguished from the virtual cut would be classified as two internal microcracks (white arrows) and one microcrack connected to a bone surface (black arrow). Figure 8 shows 3D visualizations of a detail as well as the whole bone sample subjected to IGFA that was fatigued prior to compressing to $1 \%$ and $2 \%$ strain. Interestingly no crack formation could be observed directly after subjecting the sample to the dynamic displacement cycles. The structure of the bone has changed since the elastic modulus of the sample decreased by about $10 \%$. It might well be that most of the cracks seen upon compressing the sample up to $2 \%$ strain were already there or at least initiated but were beyond the spatial resolution of the acquired tomogram. The sample visualized in Figure 8 fails in a burst-like fashion, in contrast to the band-like failure of the sample that was not fatigued. 


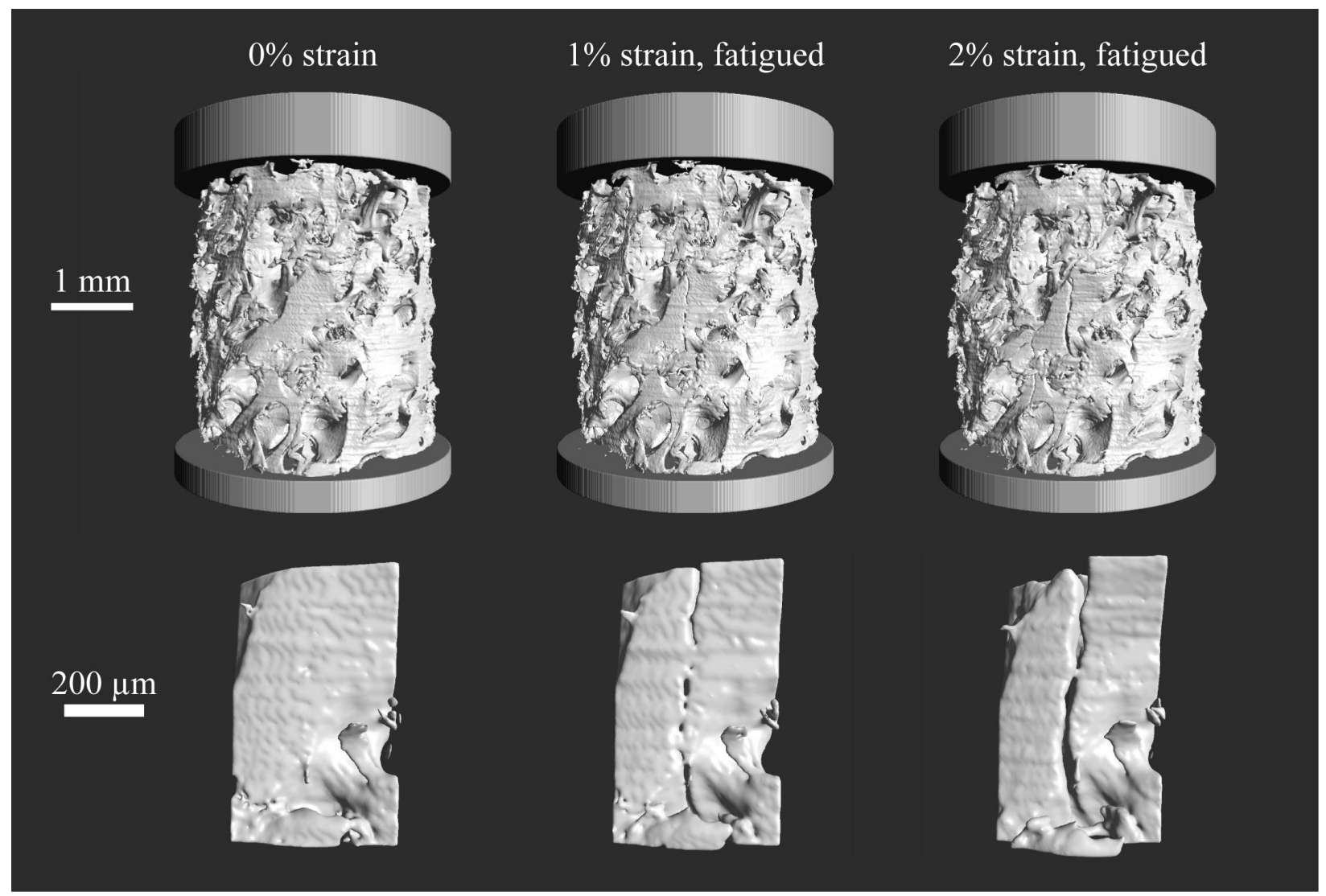

Figure 8: 3D visualization of a detail and the whole fatigued bone sample (sample B) before and after fatigue at different strains. The sample shows a burst-like fracture behavior. The detail shows the microcrack located in the middle of the 3D visualization of the whole sample. Even at $2 \%$ strain the two crack surfaces are not completely disrupted but are still connected, presumably by bone ligaments (uncracked ligament bridging). Data acquired at the MS beamline (SLS) at the photon energy of $20 \mathrm{keV}$.

It can be seen that the two pieces, that seem to shear off from each other are not fully separated even at $2 \%$ global strain. Filamentous features can be seen to span the crack. This finding is an indication for un-cracked ligament bridging, which was so far only observed in SEM images of bone (43). In terms of the detected crack volume the majority of the crack volume $(99.99 \%)$ is actually connected to the bone surface. Both samples display an increase in crack volume with increasing strain with the increase in crack volume being almost twice as high in the fatigued sample compared to the non-fatigued sample. The result of the crack detection algorithm is summarized in Table 4 for both samples. The fact that the values for internal crack and surface connected crack volumes are higher at zero strain than at $1 \%$ strain for the non-fatigued sample can be explained by a worse SNR in the data acquired at zero strain, which results in a more rippled surface and thus also a higher number for the detected crack volume.

Table 4: Quantified crack volume in $\mathrm{mm}^{3}$ applying the crack detection algorithm with 7 dilation and 8 erosion steps, resulting in a maximum detectable crack thickness of about $100 \mu \mathrm{m}$.

\begin{tabular}{|l||c|c|}
\hline \multicolumn{1}{|c|}{ Sample / Description } & Sample A (not fatigued) & Sample B (fatigued) \\
\hline \hline Internal cracks at $0 \%$ strain & 0.007 & 0.002 \\
\hline Internal cracks at $1 \%$ strain & 0.002 & 0.003 \\
\hline Internal cracks at 2\% strain & 0.002 & 0.005 \\
\hline Surface connected cracks at 0\% strain & 1.280 & 0.976 \\
\hline Surface connected cracks at 1\% strain & 1.196 & 1.010 \\
\hline Surface connected cracks at 2\% strain & 1.539 & 1.490 \\
\hline
\end{tabular}




\section{DISCUSSION}

The results from stained cell cultures show that staining in combination with SR $\mu \mathrm{CT}$ in absorption contrast mode is feasible for 3D cell culture visualization. The comparison with the unstained cell cultures demonstrates that although quantitative phase contrast SR $\mu \mathrm{CT}$ might be a feasible tool for 3D characterization making the staining procedure unnecessary, the direct comparisons of the projections and 3D visualizations of cell cultures retrieved from SR $\mu \mathrm{CT}$ to SEM micrographs underline the feasibility of the technique for hydrated biological systems at the length scale of about $1 \mu \mathrm{m}$. Depending on cell density, even the shape of individual cells can be resolved. The retrieved segmented volumes of the two cell-seeded yarns allow the quantitative characterization of the selected cell culture. The morphology evolving from different cell phenotypes is clearly accessible. Since the whole 3D dataset is available, a region of interest can be extracted and observed from any desired direction, as given in Figure 3, where the cell shape looks reasonable. Cells form complex systems (cultures) depending on their role in the natural environment. Thus, the HFFs encapsulate the scaffold recognized as a foreign body (44). In addition the cells reduce the contact interface to the scaffold. According to the computed numbers given in Table 2, the fraction of the whole cell culture surface in contact with the yarn is only $0.7 \%$, which might be interpreted as only $1.4 \%$ of the cells making full (direct) contact with the yarn. This value must be interpreted with care. The filaments, being thicker than the cells, may have a much smoother discrete surface. The adhesive cell surface, being also filament surface, could thus be much smoother than the rest of the cell culture surface. To check this assumption we computed the adhesive surface per cell (using the estimated cell density) to be $16 \mu \mathrm{m}^{2} /$ cell. Postulating a surface of about $600 \mu \mathrm{m}^{2} /$ cell (i.e. $300 \mu \mathrm{m}^{2}$ adhesive surface per cell) means that about $5 \%$ of the cells are in contact with the yarn. This estimation shows that the order of magnitude of the retrieved value is indeed reasonable. The behavior is totally different for the MC3T3 cells, which seem to grow, lining the filaments of the yarn (cp. Figure 3). This morphology can be explained by the natural function of osteoblast cells, which line the bone surface $(1,45)$. In this case the fraction of adhesive cell surface is with $14.5 \%$ two orders of magnitude higher than for the HFF cells and suggests that around $30 \%$ of the cells make full (direct) contact with the yarn. The estimation based on the adhesive surface per cell - resulting in a value of $300 \mu \mathrm{m}^{2}$ per cell - suggests, that $97 \%$ of the cells are in contact with the yarn. Thus the absolute values of aCS/CS must be treated with care.

It should be noted that only a few percent of the HFF cells are sufficient to create mechanical anchorage and stability of the entire cell culture to the yarn scaffold. Moreover, the morphology can be described in terms of the spatial cell distribution, namely the cell volume as a function of distance to the nearest filament, displayed in Figure 4. The MC3T3 cells are forming a rather homogenous distribution along the filaments. The cells are distributed in a well defined distance to the filament surface characterized by one peak in the curve of Figure 4. The HFFs show a rather inhomogeneous distribution resulting in the broad curve of Figure 4. The cells have a larger mean distance to the filament surfaces.

The mechanical tests performed with the aluminum samples show that the IMCSD device has a considerable compliance. Using the stress-strain data from the aluminum samples from both the IMCSD and the conventional testing machine the date for the bone samples acquired with the IMCSD are calibrated. The systematic error without this calibration is about $-10 \%$ for the elastic modulus of the bone samples used. The IMCSD was designed and built in order to allow IGFA experiments at the synchrotron radiation source, including cyclic loading of biological systems in a well defined hydrated environment. The constraints for the realization of such an instrument include limitations on the weight of the device since it must be carried by the high-precision sample manipulator stage. Similarly the outer geometry is constrained since the device must fit onto the stage bringing the sample into the beam and rotating it during a tomography scan. In order to have displacement control with the possibility to apply dynamic loads a piezoelectric actuator with large displacement has been chosen for the displacement exerting part. Due to the fact that the sample chamber must be X-ray translucent at the location of the sample a plastic tube has been used. The inner part of the IMCSD can rotate via the bearing connecting it to the fixed outer part, which ensures the mechanical stability of the device. Using the device compliance for the calibration of the data, correct stress-strain curves are computed as proven by the average curves presented in Figure 5. No preconditioning was performed prior to the mechanical tests on the bovine bone cylinders, since it was the intention to perform mechanical tests on the samples without changing them prior to the tests. Although it is known that the preconditioning can reduce the toe regions observed in stress-strain curves of trabecular bone specimens we suggest that leaving this part out gives a more realistic picture of the mechanical properties. Moreover, it is questionable if the preconditioning is also applicable for such small samples as investigated here. In order to compute meaningful mechanical parameters we corrected the data by the use of the regression lines fitted for the elastic moduli in the individual curves. The point of zero strain was set at the intersection of each regression line with zero stress. It can be seen that the parameters derived from the mechanical tests from the 
two different machines in this way are in agreement with each other. The relatively high coefficients of variation are presumably due to the rather small sample size and limited number of samples. As already mentioned, not correcting the stain values would result in an elastic modulus about $10 \%$ below the actual one. As the bone samples were rather stiff, only $20 \%$ less stiff than trabecular whale bone (21), they represent an upper threshold for the properties of bone samples to be investigated with IMCSD. The bone samples measured with IGFA show good agreement between the input and real displacement, as the recorded forces were rather low compared to the samples investigated for mechanical testing.

Comparing the results from the two specimens subjected to IGFA it was shown that the fatigued sample (B) failed in a different manner than the non-fatigued sample (A). It bursts and shows a failure band (cp. Figures 6 and 8). This finding is, however, only an indication since just two samples were investigated. Both samples failed between $1 \%$ and $2 \%$ strain. The development of crack volume is different for both samples. In the fatigued case the total crack volume increase between zero and $2 \%$ strain is about $0.5 \mathrm{~mm}^{3}$, whereas in the non-fatigued case the total volume increases by only about $0.3 \mathrm{~mm}^{3}$. It must of course be noted that the crack surface would be a more useful parameter since it directly relates to the energy dissipation.

Overall the 3D visualization of the cracks, i.e. microcracks and microfractures, without staining is a breakthrough. For the first time these features can be non-destructively detected in a 3D manner (cp. Figures 6-8). The result shows that the majority of the detected cracks are connected to the bone surface, suggesting that "internal" cracks frequently reported in histological post-hoc analyses of microdamage are rather an artifact emerging from the technique used, rather than a true finding. Interestingly, a considerable number of cracks are per definition microfractures, since they extend through an entire trabecula. Looking at the virtual sections of such microfractures, these were classified as microcracks (cp. Figure 7). Thus the classification of a microfracture and a microcrack from the 2D image is ambiguous, depending on the location and the direction of a given section, a microfracture might be classified correctly or not. Moreover, the diffuse, crosshatched, and confined cracks found in the $2 \mathrm{D}$ analyses can also be explained by the limited assessment of the cracks using the conventional techniques. Despite the fact that the used detection algorithm for crack detection is not devoid of errors so far the surface-connected crack volume does also increase much more than the internal cracks. Since the baseline of cracks, due to detected artifacts, can be assumed to stay more or less constant this is an indication that the amount of surface-connected cracks is much higher than the number of internal cracks. The crack detection algorithm must be further developed in order to exclude artifacts to give a clear picture of the evolution of microcracks and microfractures. Then also the crack surface can be quantified in a 3D manner. The fact that an indication of un-cracked ligament bridging can be seen is surprising and has to be validated with LM or SEM techniques. This makes the technique as a whole even more promising. Since the effect is known to increase the fracture toughness IGFA using SR $\mu \mathrm{CT}$ might be a way to study the effect in 3D, relating it to healthy or diseased bone status. Besides being a toughening mechanism the un-cracked ligament bridging might be moreover an effect needed for bone repair by osteoblasts and osteoclasts as it provides information of broken bone pieces that need to be mended together. If the organic matrix of bone changes due to disease it might be that the absence of such ligaments prevents the correct repair of bone microarchitecture and thus trabeculae are irrecoverably lost.

\section{CONCLUSION}

In this review we present procedures for improved visualization of hard and soft biological tissues. Besides the power of SR $\mu \mathrm{CT}$ for cell culture and single cell imaging, we have presented a geometry-based algorithm for differentiation between the two phases in our cell- yarn model system. The segmentation allows quantification of the relative cell density in a spatially resolved manner and shows its link to the yarn geometry. The cell cultures of the two cell types HFF and MC3T3 create different morphologies on the yarn. The morphology can be associated with cell function in the natural environment of these cells: fibroblasts encapsulated the yarn, whereas the osteoblast-like cells lined it. Interestingly also the contact area of the fibroblasts is small compared to the osteoblast-like cells. Only $1 \%$ to $5 \%$ of the HFF cells are actually in contact with a filament surface. The osteoblast-like cells, where $30 \%$ to $100 \%$ seem to have contact with the yarn, exhibit the opposite behavior. The results show that SR $\mu \mathrm{CT}$ does not only give qualitative information of cell cultures, but also provides the full 3D quantification of the sample phases, allowing statements about cell distribution, cell culture morphology, cell adhesion as well as correlations to 3D scaffold geometry. Therefore, SR $\mu \mathrm{CT}$ can provide truly complementary data to state of the art investigations on cells in an opaque 3D environment. Moreover, such single samples can be divided into several sub-samples for analysis: the quantitative assessment of several yarn sections separated by some distance allows even for sufficient statistics to compare different 
groups. In such a manner experiments comparing different influences on cell cultures such as toxins or growth factors could be tested in a fully quantitative 3D manner.

The IMCSD device was successfully installed and utilized at the MS beamline (SLS) at the PSI. Besides being an IGFA device it was also validated with comparative mechanical tests performed on the IMCSD and a conventional mechanical testing device using solid aluminum and trabecular bovine bone cylinders both $4 \mathrm{~mm}$ in height and $3.7 \mathrm{~mm}$ in diameter. The results from the aluminum give the compliance for the IMCSD to be $(0.13+/-0.02) \mu \mathrm{m} / \mathrm{N}$. Using this value for calibrating the measured strain gives comparable stress-strain curves as retrieved from a commercial testing device. This was shown for the trabecular bovine bone samples subjected to mechanical testing, which gave very similar values for the mechanical parameters of elastic modulus, stiffness, yield strain, and strength, as well as ultimate compressive stress and strain, respectively. We estimate that without the correction the systematic error for these values is around $-10 \%$. Measurements on less compliant samples will give even lower errors and the error can be corrected by a calibration measurement. Moreover, during IGFA recorded projections will give an even better idea of the applied strains as they can be directly calculated from these. The acquired results from IGFA prove that the IMCSD device can be successfully used for this purpose. The stability of the applied displacement is given, as no artifacts due to sample movement during the scans could be seen. The results obtained from IGFA give indications of the differences between failure of a non-fatigued and a fatigued sample. These indications state that the failure behavior is different and that quantitatively the crack volume encountered is larger in the fatigued case. Moreover, the uncovered details, microcracks and microfractures underline the power of IGFA and SR $\mu \mathrm{CT}$ as tools to investigate bone failure behavior. The results found shed new light on microcrack investigations and suggest that the classification of cracks in $2 \mathrm{D}$ analyses is ambiguous. The definitions of microcracks and microfractures should be renewed in this context. The majority of detected crack volume is connected to the bone surface and only a very small amount of crack volume is classified as internal cracks. This result is consistent with the strain based failure mode for compact bone found by Nalla et al. (43) and gives rise to the assumption that the microcracks generally initiate at a trabecular bone surface. The results even give an indication of un-cracked ligament bridging. If this result can be validated, SR $\mu \mathrm{CT}$ might even be used to study toughening mechanisms and the ion-mediated repair process that is associated with them.

In Summary, more biologically oriented experiments are now to be done for the developed techniques presented in this review for further validation.

\section{ACKNOWLEDGEMENT}

The authors thank Martin Müller of ETH Zürich for SEM sample preparation and Rhiannon Evison for help during bone preparation. We are gratefully indebted to the Swiss National Science Foundation for financial support of this project (Grant No. 2153-057127.99) and to HASYLAB/DESY (II-99-077) and ESRF (LS-1980) and SLS/PSI for beamtime.

\section{REFERENCES}

1. Alberts, B., Bray, D., Lewis, J., Raff, M., Roberst, K. \& Watson, J. D. (1994) Molecular Biology of the Cell (Garland Publishing, New York).

2. Abbott, A. Cell culture: biology's new dimension (2003) Nature 424, 870-2.

3. Gibson, S. F. \& Lanni, F. Experimental Test of an Analytical Model of Aberration in an Oil-Immersion Objective Lens Used in 3-Dimensional Light-Microscopy (1991) Journal of the Optical Society of America aOptics Image Science and Vision 8, 1601-1613.

4. McNally, J. G., Preza, C., Conchello, J. A. \& Thomas, L. J. Artifacts in Computational Optical-Sectioning Microscopy (1994) Journal of the Optical Society of America a-Optics Image Science and Vision 11, 10561067.

5. Marincek, B., Ros, P. R., Reiser, M. \& E., B. M. (2000) Multislice CT - A Practical Guide (Springer, Berlin, Germany.

6. Weiss, D., Schneider, G., Niemann, B., Guttmann, P., Rudolph, D. \& Schmahl, G. Computed tomography of cryogenic biological specimens based on X-ray microscopic images (2000) Ultramicroscopy 84, 185-97.

7. Ray, N. F., Chan, J. K., Thamer, M. \& Melton, L. J., 3rd Medical expenditures for the treatment of osteoporotic fractures in the United States in 1995: report from the National Osteoporosis Foundation (1997) $J$ Bone Miner Res 12, 24-35.

8. Cooper, C., Atkinson, E. J., Jacobsen, S. J., O'Fallon, W. M. \& Melton, L. J., 3rd Population-Based Study of Survival after Osteoporotic Fractures (1993) Am J Epidemiol 137, 1001-5.

9. McElhaney, J. H., Fogle, J. L., Melvin, J. W., Haynes, R. R., Roberts, V. L. \& Alem, N. M. Mechanical properties of cranial bone (1970) J Biomech 3, 495-511. 
10. Carter, D. R. \& Hayes, W. C. The compressive behavior of bone as a two-phase porous structure (1977) Journal of Bone and Joint Surgery [Am] 59, 954-962.

11. Williams, J. L. \& Lewis, J. L. Properties and an anisotropic model of cancellous bone from the proximal tibial epiphysis (1982) J Biomech Eng 104, 50-56.

12. Rice, J. C., Cowin, S. C. \& Bowman, J. A. On the dependence of the elasticity and strength of cancellous bone on apparent density (1988) Journal of Biomechanics 21, 155-168.

13. Odgaard, A. \& Linde, F. The underestimation of Young's modulus in compressive testing of cancellous bone specimens (1991) Journal of Biomechanics 24, 691-698.

14. Keller, T. S. Predicting the compressive mechanical behavior of bone (1994) J Biomech 27, $1159-68$.

15. Keaveny, T. M., Guo, X. E., Wachtel, E. F., McMahon, T. A. \& Hayes, W. C. Trabecular bone exhibits fully linear elastic behavior and yields at low strains (1994) Journal of Biomechanics 27, 1127-36.

16. Ciarelli, M. J., Goldstein, S. A., Kuhn, J. L., Cody, D. D. \& Brown, M. B. Evaluation of orthogonal mechanical properties and density of human trabecular bone from the major metaphyseal regions with materials testing and computed tomography (1991) J Orthop Res 9, 674-82.

17. Burr, D. B., Martin, R. B., Schaffler, M. B. \& Radin, E. L. Bone remodeling in response to in vivo fatigue microdamage (1985) J Biomech 18, 189-200.

18. Burr, D. B., Forwood, M. R., Fyhrie, D. P., Martin, R. B., Schaffler, M. B. \& Turner, C. H. Bone microdamage and skeletal fragility in osteoporotic and stress fractures (1997) J Bone Miner Res 12, 6-15.

19. Tantillo, M. \& Müller, R. Image-Guided Failure Assessment of Porous Materials (2000) in Material Research Society Symp. Proc., pp. 25 - 30.

20. Muller, R., Gerber, S. C. \& Hayes, W. C. Micro-compression: a novel technique for the nondestructive assessment of local bone failure (1998) Technol Health Care 6, 433-44.

21. Nazarian, A. \& Muller, R. Time-lapsed microstructural imaging of bone failure behavior (2004) J Biomech 37, 55-65.

22. O'Brien, F. J., Taylor, D., Dickson, G. R. \& Lee, T. C. Visualisation of three-dimensional microcracks in compact bone (2000) Journal of Anatomy 197, 413-420.

23. Schaffler, M. B., Pitchford, W. C., Choi, K. \& Riddle, J. M. Examination of compact bone microdamage using back-scattered electron microscopy (1994) Bone 15, 483-8.

24. Thurner, P., Muller, B., Beckmann, F., Weitkamp, T., Rau, C., Muller, R., Hubbell, J. A. \& Sennhauser, U. Tomography studies of human foreskin fibroblasts on polymer yarns (2003) Nuclear Instruments \& Methods in Physics Research Section B-Beam Interactions with Materials and Atoms 200, 397-405.

25. Hildebrand, T. \& Ruegsegger, P. Quantification of bone microarchitecture with the structure model index (1997) Comput Methods Biomech Biomed Engin 1, 15-23.

26. Stampanoni, M., Borchert, G., Wyss, P., Abela, R., Patterson, B., Hunt, S., Vermeulen, D. \& Ruegsegger, P. High resolution X-ray detector for synchrotron-based microtomography (2002) Nuclear Instruments \& Methods in Physics Research Section a-Accelerators Spectrometers Detectors and Associated Equipment 491, 291-301.

27. Beckmann, F., Bonse, U. \& Biermann, T. New developments in attenuation- and phase-contrast microtomography using synchrotron radiation with low and high photon energies (1999) in Proceedings of SPIE, ed. Bonse, U., San Diego), Vol. 3772, pp. 179-187.

28. Grodzins, L. Critical Absorption Tomography of Small Samples - Proposed Applications of Synchrotron Radiation to Computerized-Tomography .2. (1983) Nuclear Instruments \& Methods in Physics Research 206, 547-552.

29. Müller, B., Thurner, P., Beckmann, F., Weitkamp, T., Rau, C., Bernhardt, R., Karamuk, E., Eckert, L., Buchloh, S., Wintermantel, E., Scharnweber, D. \& Worch, H. (2002) in Developments in X-ray tomography III, ed. Bonse, U., Vol. 4503, pp. 178-188.

30. Thurner, P., Muller, B. \& Beckmann, F. An optimization procedure for spatial and density resolution in hard X-ray micro-computed tomography (2004) Nuclear Instruments \& Methods in Physics Research Section BBeam Interactions with Materials and Atoms in press.

31. Weitkamp, T., Rau, C., Snigirev, A. A., Benner, B., Günzler, T. F., Kuhlmann, M. \& Schroer, C. G. In-line phase contrast in synchrotron-radiation microradiography and tomography (2002) in Developments in X-ray Tomography III, ed. Bonse, U. (SPIE, San Diego), Vol. 4503, pp. 92-102.

32. Thurner, P., Muller, B., Sennhauser, U., Hubell, J. A. \& Muller, R. Tomography Studies of Biological Cells on Polymer Scaffolds (2004) Journal of Physics: Condensed Matter 16, S3499-3510. 
33. Suzuki, K., Horiba, I. \& Sugie, N. Linear-time connected-component labeling based on sequential local operations (2003) Computer Vision and Image Understanding 89, 1-23.

34. Haralick, R. M., Sternberg, S. R. \& Zhuang, X. H. Image-Analysis Using Mathematical Morphology (1987) Ieee Transactions on Pattern Analysis and Machine Intelligence 9, 532-550.

35. Cloetens, P., Ludwig, W., Baruchel, J., Van Dyck, D., Van Landuyt, J., Guigay, J. P. \& Schlenker, M. Holotomography: Quantitative phase tomography with micrometer resolution using hard synchrotron radiation $\mathrm{x}$ rays (1999) Applied Physics Letters 75, 2912-2914.

36. Thurner, P. Imaging of Cellular and Extra-Cellular Stressed Matter Using Synchrotron Radiation Based Micro-Computed Tomography (2004) in Phd Thesis in Materials Science (Swiss Federal Institue of Technology, Zürich), Vol. 15535, pp. 243.

37. Lorensen, W. E. \& Cline, H. E. Marching Cubes: A High Resolution 3D Surface Construction Algorithm (1987) Computer Graphics 21, 163-169.

38. Fyhrie, D. P. \& Schaffler, M. B. Failure mechanisms in human vertebral cancellous bone (1994) Bone 15, 1059.

39. Wachtel, E. F. \& Keaveny, T. M. Dependence of trabecular damage on mechanical strain (1997) J Orthop Res 15, 781-7.

40. Fazzalari, N. L., Forwood, M. R., Smith, K., Manthey, B. A. \& Herreen, P. Assessment of cancellous bone quality in severe osteoarthrosis: bone mineral density, mechanics, and microdamage (1998) Bone 22, 381-8.

41. O'Brien, F. J., Taylor, D. \& Lee, T. C. Microcrack accumulation at different intervals during fatigue testing of compact bone (2003) Journal of Biomechanics 36, 973-980.

42. Lee, T. C., Arthur, T. L., Gibson, L. J. \& Hayes, W. C. Sequential labelling of microdamage in bone using chelating agents (2000) Journal of Orthopaedic Research 18, 322-325.

43. Nalla, R. K., Kinney, J. H. \& Ritchie, R. O. Mechanistic fracture criteria for the failure of human cortical bone (2003) Nat Mater 2, 164-8.

44. Sanders, J. E. \& Rochefort, J. R. Fibrous encapsulation of single polymer microfibers depends on their vertical dimension in subcutaneous tissue (2003) Journal of Biomedical Materials Research Part A 67A, 1181-1187.

45. Menton, D. N., Simmons, D. J., Chang, S. L. \& Orr, B. Y. From bone lining cell to osteocyte--an SEM study (1984) Anatomical Record 209, 29-39. 